\title{
An Egr-1 master switch for arteriogenesis: Studies in Egr-1 homozygous negative and wild-type animals
}

\author{
Cristian Sorin Sarateanu, MD, ${ }^{a}$ Mauricio A. Retuerto, BS, ${ }^{a}$ James T. Beckmann, BS, ${ }^{a}$ Leslie McGregor, BS, ${ }^{a}$ \\ JoAnn Carbray, BS, ${ }^{a}$ Gerald Patejunas, PhD, ${ }^{b}$ Lina Nayak, ${ }^{a}$ Jeffrey Milbrandt, MD, ${ }^{c}$ and Todd K. Rosengart, $\mathrm{MD}^{\mathrm{b}}$
}

From Evanston Northwestern Healthcare, Evanston, Ill ${ }^{\text {a }}$; Evanston Northwestern Healthcare, Evanston, Ill, and Feinberg School of Medicine of Northwestern University, Chicago, Ill'b; and Washington University, St Louis, Mo. ${ }^{c}$

Supported in part by the National Institute of Health/National Heart, Lung, and Blood Institute (R01HL66981 and R01HL57318) and Evanston Northwestern Healthcare Women's Auxiliary Fund (Evanston, Ill).

Read at the Eighty-fifth Annual Meeting of The American Association for Thoracic Surgery, San Francisco, Calif, April 10-13, 2005 .

Received for publication April 11, 2005; revisions received July 12 , 2005; accepted for publication Aug 2, 2005.

Address for reprints: Todd K. Rosengart, MD, 2650 Ridge Ave, Burch 100, Evanston, IL 60201 (E-mail: trosengart@ enh.org).

J Thorac Cardiovasc Surg 2006;131:138-45

$0022-5223 / \$ 32.00$

Copyright $\odot 2006$ by The American Association for Thoracic Surgery

doi:10.1016/j.jtcvs.2005.08.052
Background: Arteriogenesis has been implicated as an important biologic response to acute vascular occlusion. The early growth response 1 (Egr-1) gene encodes an immediate-early response transcription factor that is upregulated by changes in vascular strain and that in turn upregulates a number of putative angiogenic and arteriogenic growth factors. We therefore hypothesized that early growth response 1 might be a critical arteriogenic messenger that induces revascularization in the setting of acute vascular occlusions.

Methods: Wild-type or Egr-1 ${ }^{-1-}$ (null) C57 BL mice, or Sprague-Dawley rats, underwent unilateral iliofemoral artery excision and subsequent analyses for angiogenesis and arteriogenesis through cell-specific immunohistochemistry. Rats were also administered an adenoviral vector encoding for Egr-1 (AdEgr group), noncoding vectors (AdNull group), or saline, after which these animals were assessed by means of serial laser Doppler perfusion imaging and morphologic examination of rat foot-pad ischemic lesions.

Results: Egr-1 wild-type mice demonstrated an equivalent number of capillaries but a greater number of arterioles following excision versus Egr-1 null mice. AdEgr group rats demonstrated greater distal perfusion from 7 to 21 days after excision compared with control animals $(P<.02)$, which approximated normal perfusion at 21 days after excision. AdEgr group rats also demonstrated greater arteriolar density and less severe ischemic foot-pad lesions than control animals.

Conclusion: These data suggest the importance of Egr-1 as a critical and potentially therapeutic mediator of revascularization after vascular occlusion and implicate arteriogenesis (collateral vessel formation) as a critical component of this process.

$\mathrm{R}$ ecognition of the central role played by angiogenic growth factors in the endogenous processes of tissue vascularization has led to the development of a strategy of therapeutic angiogenesis, wherein exogenous growth factors are administered to ischemic tissues to enhance the reperfusion of these tissues. ${ }^{1-7}$ Recent evidence suggests, however, that the administration of angiogenic mediators, such as vascular endothelial growth factor (VEGF), that might primarily induce capillary formation might also not be sufficient to induce stable neovascularization or to induce collateral vessel formation or arteriogenesis. ${ }^{8-13}$ Moreover, because the development of a mature and robust vascular network would seem to optimally require a precise spatial and temporal regulation of a large number of angiogenic stimulators, arteriogenic stimulators, or both and cofactors, it might be preferable, as opposed to the prevalent current practice of administering isolated growth factors, to deliver a transcription factor that upregulates the downstream expression of the various growth factors as would occur under endogenous regulatory mechanisms.

The early growth response (Egr) 1 transcription factor (also known as zif 268, TIS 8, nerve growth factor induced A, and Krox 24) is an 80- to 82-kd, 533-amino-acid 


\section{Abbreviations and Acronyms \\ AdEgr-1 = adenoviral vector encoding for Egr-1 \\ AdNull = noncoding vector \\ Egr-1 = early growth response transcription factor 1 \\ LDPI = laser Doppler perfusion imaging \\ PBS = phosphate-buffered saline \\ ROI = region of interest \\ SMA $=$ smooth muscle actin \\ VEGF = vascular endothelial growth factor}

residue protein that is the prototypical member of the immediate-early gene family of zinc-finger transcription factors that includes Egr-2, Egr-3, and Egr-4. ${ }^{14,15}$ Expression of this pluripotent transcription factor is modulated by changes in vascular wall shear stress that would occur in the setting of an acute vascular occlusion and directly or indirectly results in the upregulated expression of numerous growth factors implicated in both angiogenesis and arteriogenesis. ${ }^{16-19}$ Egr-1 is thus an intriguing potential mediator of revascularization responses to acute vascular occlusions. In this regard we recently demonstrated that Egr-1 null (knockout) mice subjected to femoral artery excision demonstrate a nearly complete absence of revascularization competency. ${ }^{20} \mathrm{We}$ therefore hypothesized and now report data demonstrating a deficiency in arteriogenesis in Egr-1 null animals and that the therapeutic administration of Egr-1 in wild-type animals is capable of inducing a robust revascularization in the setting of acute vascular occlusion.

\section{Materials and Methods}

\section{Egr-1 Knockout Animals}

A colony of Egr-1 ${ }^{-1-}$ (null) C57 BL/6 mice (gift of Dr Jeffrey Milbrandt, Washington University, St Louis, Mo.) ${ }^{21,22}$ was established at the Coon Research Center at Evanston Northwestern Healthcare by using standard breeding procedures. Animal handling at all times was in accordance with institutional animal care and use committee guidelines. Animals selected from this colony underwent genotyping at 25 days after birth, as previously described, ${ }^{20}$ after which Egr-1 null or wild-type animals underwent experimental studies at approximately 2 to 4 months of age.

\section{Gene Transfer Vectors}

The Egr-1 transfer vector (adenoviral vector encoding for Egr-1 [AdEgr]) used in these studies was an E1a ${ }^{-}$, partial E1b ${ }^{-}$, partial E3 ${ }^{-}$ adenovirus vector based on human adenovirus serotype 5 containing the cytomegalovirus early promoter-enhancer driving the complementary DNA for Egr-1, which was prepared as previously described (gift of J. Milbrandt). ${ }^{23,24}$ As a negative control, a similar adenovirus containing no gene in the expression cassette (AdNull) was used. Vector competency was assessed on HT1080 cells (American Type Culture Collection no. CCl-121), which were treated with 50 vector pu/cell. Cells were harvested into Trizol Reagent (Invitrogen, Carlsbad, Calif), and RNA was purified, reverse transcribed, and subjected to semiquantitative polymerase chain reaction by using rat Egr-1 primers (GenBank accession no. NM_012551). ${ }^{20}$

\section{Vascular Excision in Mice}

Wild-type or homozygous Egr-1-deleted (null) mice (25 g) selected from the C57 BL/6 mouse colony were anesthetized with pentobarbital (60 mg/kg administered intraperitoneally) after induction with a mixture of isoflurane $(3.5 \mathrm{~L} / \mathrm{min})$ and $100 \%$ oxygen $(3 \mathrm{~L} / \mathrm{min})$. A midline abdominal incision was then made, and the proximal end of the femoral artery at the inguinal ligament and the distal portion of the saphenous artery were ligated with 4-0 silk sutures. The artery and all side branches were then dissected free, and this entire vascular segment was removed (type I preparation). ${ }^{25}$ The incision was then closed, and the animals were recovered as per standard protocols.

\section{Adenoviral Vector Injection in Rats}

For therapeutic studies, male Sprague-Dawley rats (250-320 g; Harlan Sprague Dawley, Inc, Indianapolis, Ind) treated in accordance with institutional care and use committee guidelines underwent anesthetic induction with a mixture of ketamine $(80 \mathrm{mg} / \mathrm{kg}$ administered intraperitoneally) and xylazine (12 mg/kg administered intraperitoneally). The rats were placed in the dorsal decubitus position, and the abdomen and both inferior limbs were shaved and cleaned with povidoneiodine solution. As opposed to the type I femoral excision preparation used in mice to minimize necrosis in the interest of histologic studies, a more extensive (type II) iliofemoral excision model was created in rats to create a maximal degree of ischemia to conduct physiologic and morphologic studies of the effects of AdEgr administration on distal perfusion and revascularization. The retroperitoneal space on the left side was entered through a midline abdominal incision, and the common iliac artery was dissected free and ligated. The abdomen was closed in anatomic layers. Immediately thereafter, a left groin incision was performed, and the common, superficial, and deep femoral arteries and veins were dissected free. The common proximal, superficial, and deep femoral arteries and veins were ligated and excised, whereas the most proximal perforant branch of the common femoral artery was carefully preserved.

Six separate $20-\mu \mathrm{L}$ injections of $2 \times 10^{9}$ pu (total dose: $1.2 \times$ $10^{10} \mathrm{pu}$ diluted in $120 \mu \mathrm{L}$ of phosphate-buffered saline [PBS]) of AdEgr $(n=8)$ or AdNull $(n=7)$ or $120 \mu \operatorname{L}$ of PBS $(n=7)$ were then delivered into the adductor muscles by using the muscular branch of the common femoral artery as a landmark. The groin incision was then closed with a running suture, and the animals were allowed to recover according to standard protocols. All the procedures were performed by the same operator, and the identity of the animals was revealed only at the end of the study (postoperative day 21).

\section{Laser Doppler Perfusion}

Laser Doppler perfusion imaging (LDPI) was used to measure distal hind-limb perfusion preoperatively and at serial time points after vascular excision (immediately after surgical intervention and at postoperative days 1, 3, 7, 14, and 21). Duplicate laser Doppler perfusion images were obtained in a blinded manner by using an LDPI system (Perimed, Jarfalla, Sweden) through clipped and depilated hind-limb skin surfaces after placing the rats on a $37^{\circ} \mathrm{C}$ heating pad for 10 minutes. Regions of interest (ROIs) encompassing either the entire ligated or the contralateral nonligated hind limb were analyzed with accessory software (Patch Test) provided by the manufacturer, as previously described. ${ }^{25}$ More specifically, the ROIs were determined by using color images of the ventral surface of the hind limbs by placing a standard, computer-generated rectangle over the most distal 


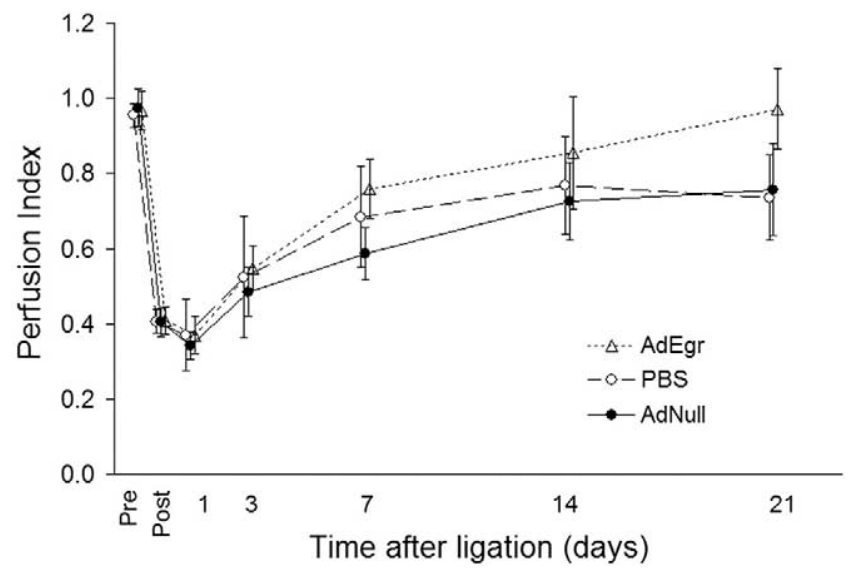

Figure 1. Quantitative analysis of perfusion index, calculated as described in the Methods section. Note near normalization (index approximately 1) of perfusion in AdEgr-treated animals at day 21. AdEgr-1, Adenoviral vector encoding for Egr-1; PBS, phosphatebuffered saline; AdNull, noncoding vector.

part of each limb, after which the system software was used to calculate a mean intensity of Doppler signal within the ROI. A perfusion index for each animal was expressed as the average of the ratios of the ROIs for the ligated versus the contralateral, nonligated limb to minimize data variables caused by ambient light and temperature.

\section{Clinical Ischemia Score}

Rats were observed for evidence of ischemic hind-limb lesions after anesthetic induction and LDPI scanning at days 7, 14, and 21 postexcision. A severity scale was established from prior standards ${ }^{26}$ on the basis of the characteristics of the lesions on the hind limbs and paws, as follows: $1+$, normal; $2+$, healed pressure sores (trace soft tissue loss without scab); $3+$, nonhealed pressure sores (partial thickness-skin loss covered with scab); $4+$, gangrene of the tips of the toes; and 5+, gangrene with amputation of phalanges-toes. Photographs (Sony Cybershot DSC P72 Camera) were taken for each animal at the intervals described, stored, and analyzed in a blinded fashion.

\section{Histology}

Animals were killed at either day 7 or day 21 after excision by means of pentobarbital overdose. The adductor musculature was excised and fixed with zinc-formalin before paraffin embedding. Sections taken at $100-\mu \mathrm{m}$ intervals with a $5-\mu \mathrm{m}$ thickness were prepared for immunohistochemical staining with avidin/horseradish peroxidase with chromogenic developing reagents and were hematoxylin and eosin counterstained. Sections were labeled with biotinylated smooth muscle actin (SMA) monoclonal antibody (Dako, Carpinteria, Calif) or biotinylated Galanthus nivalis lectin specific to rodent endothelial cells (Vector Laboratories, Burlington, Calif). SMA-positive vessels and lectin-positive vessels were counted from 6 randomly selected $(200 \times)$ fields in a blinded manner assisted by Metamorph Software (Universal Imaging, Downingtown, Pa). Specific

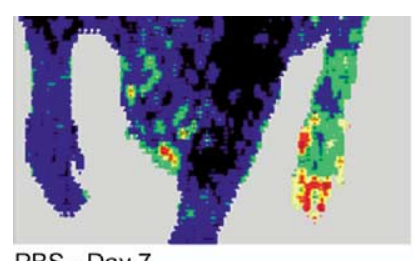

PBS - Day 7

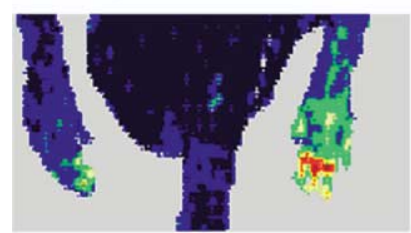

AdNull - Day 7
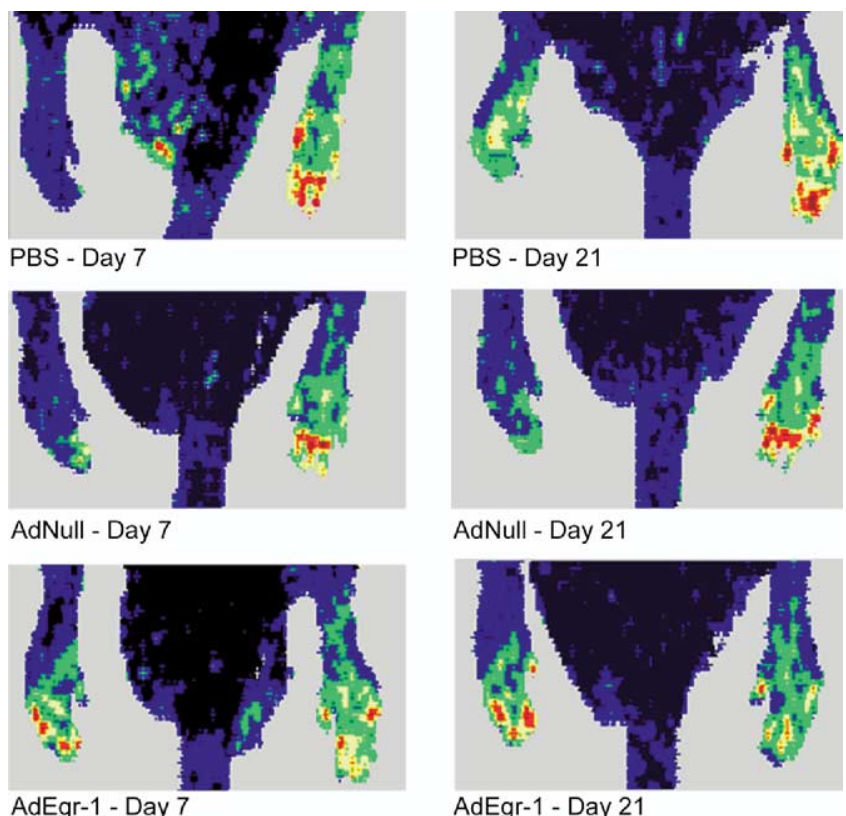

PBS - Day 21

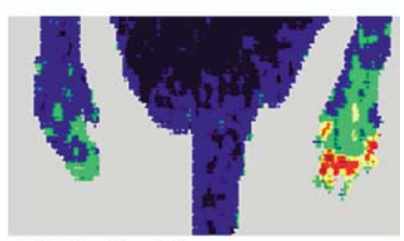

AdNull - Day 21

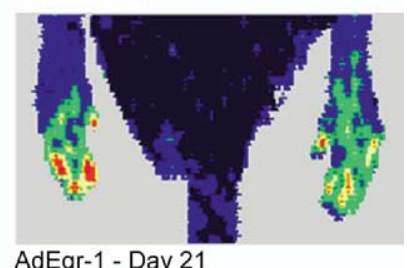

Figure 2. Laser Doppler perfusion imaging at days 7 and 21 after excision. Low or no blood perfusion is displayed as dark blue, and the highest perfusion level is displayed as red. Note early reconstitution of flow in AdEgr-1-treated animals and near equalization at day 21. PBS, Phosphate-buffered saline; AdNull, noncoding vector; AdEgr-1, adenoviral vector encoding for Egr-1.

quantification was also made of the number of vessels per field measuring $50 \mu \mathrm{m}$ or larger (arterioles). ${ }^{10}$

\section{Statistical Analysis}

Data are expressed as means and standard deviations. One-way analysis of variance or $\chi^{2}$ analysis, followed by the post hoc Bonferonni correction, was used to compare results between groups, as appropriate.

\section{Results}

\section{Arteriogenesis Deficit in Egr-1 Null Animals}

Specimens from the ischemic limbs of Egr-1 null and wild-type mice obtained 21 days after femoral artery excision (type I preparation) were assessed for evidence of arteriogenesis and angiogenesis. Egr-1 null mice demonstrated fewer arterioles per microscopic field compared with wild-type mice ( $14 \pm 5$ for Egr-1 null mice vs $22 \pm$ 2 for wild-type mice, $P=.06$ ), but an equivalent number of capillaries $(115 \pm 24$ for Egr-1 null mice vs $104 \pm 41$ for wild-type mice). The morphology of both large and small vessels was similar in null compared with wildtype animals (data not shown). 

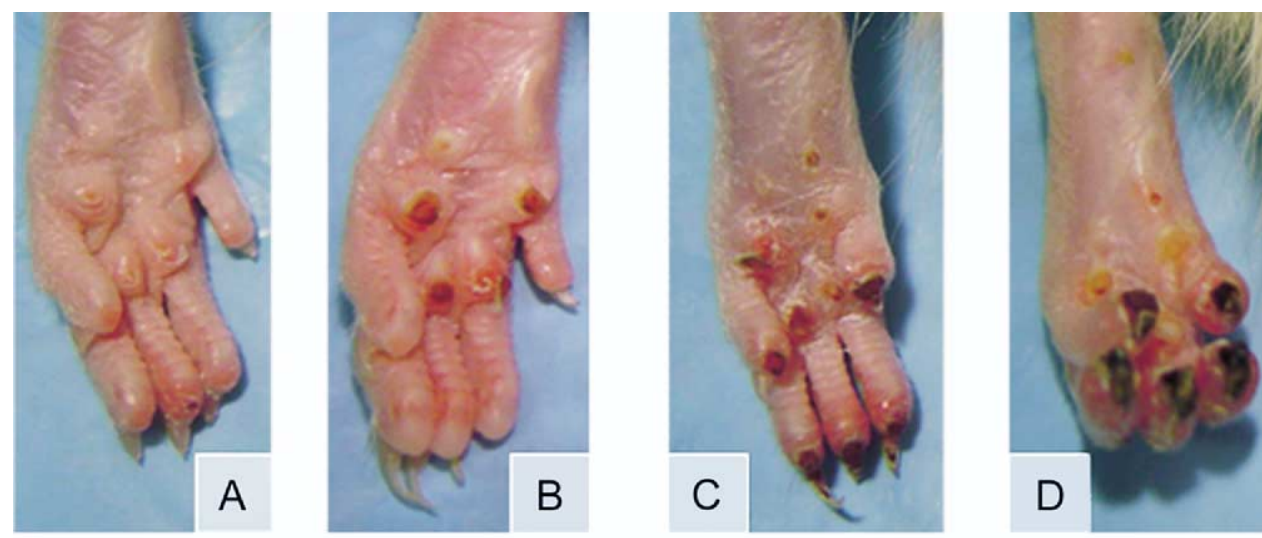

A

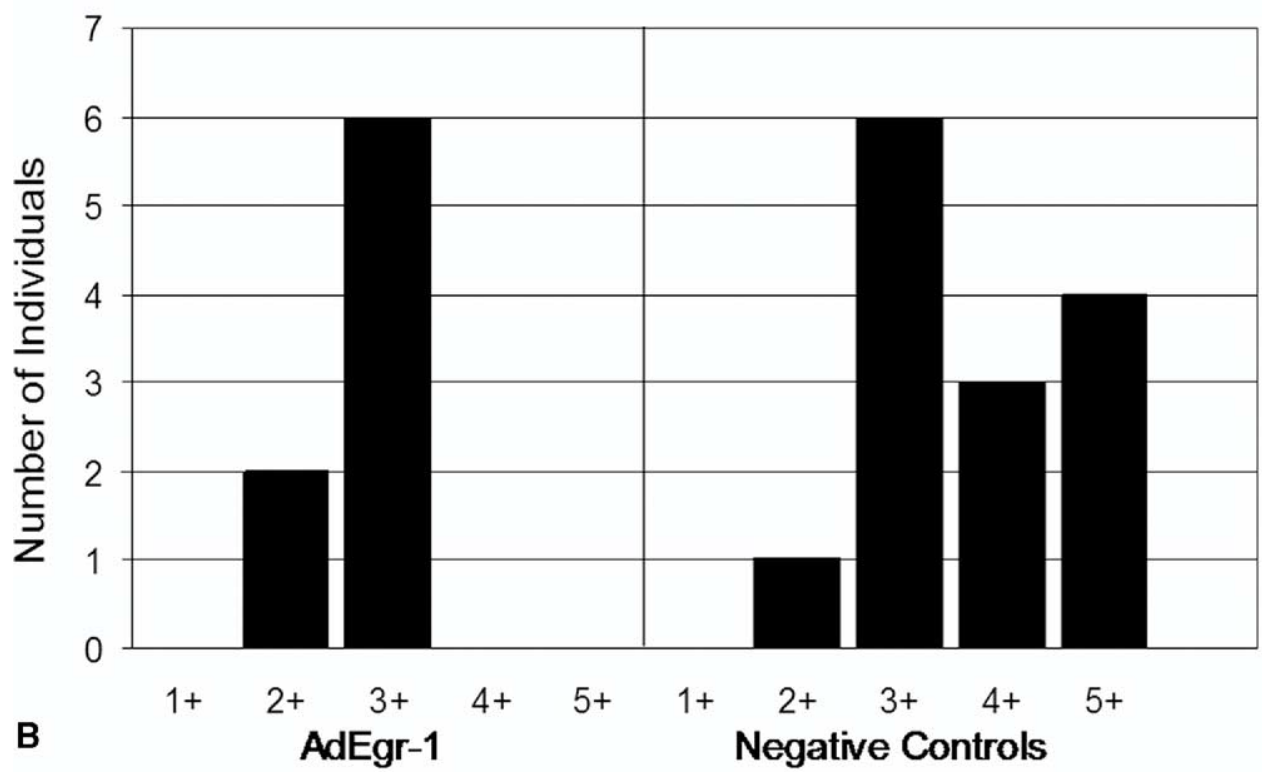

Figure 3. Top, Representative lesions as seen 21 days after excision: $a$, healed pressure sores, soft tissue loss trace without scab $(2+)$; $b$, nonhealed pressure sores, partial thickness and skin loss covered with scab $(3+)$; $c$, necrosis of the tips of the toes $(4+)$; and $d$, gangrene with amputation of phalanges-toes $(5+)$. Please see the Methods section for details. Bottom, Distribution of lesions between groups. AdEgr, adenoviral vector encoding for Egr.

\section{Laser Doppler Perfusion Analysis}

Laser Doppler perfusion analysis was performed on rats having undergone iliofemoral artery excision (type II preparation) and administration of AdEgr, AdNull, or PBS. The ligated/nonligated hind-limb perfusion index was equivalent (approximately 1) at baseline in all groups (Figure 1). Immediately after vascular excision, the ratio of ligated/nonligated hind-limb perfusion decreased to a similar extent in all groups (range, 0.34-0.38). The recovery of limb perfusion accelerated in a first phase between days 3 and 7 after excision, with the steepest slope demonstrated by the AdEgr-1-treated group when compared with the PBS- and AdNull-treated groups (day 7 perfusion index: $0.76 \pm 0.08$ for AdEgr vs $0.68 \pm 0.13$ for PBS and $0.58 \pm 0.07$ for AdNull, $P=$
$.01)$. Although the rate of improvement in perfusion slowed thereafter, AdEgr-1-treated animals demonstrated comparatively greater distal perfusion of the ligated versus the nonligated hind limb than did control animals at day 21 after excision (Figure 2). Distal perfusion in AdEgr-treated animals reapproximated baseline values, which was significantly greater than that seen in control animals (day 21 perfusion index: $0.97 \pm 0.1$ for AdEgr1-treated animals vs $0.73 \pm 0.11$ for PBS-treated animals and $0.75 \pm 0.12$ for AdNull-treated animals, $P<.006$ ).

\section{Clinical Ischemia Score}

Footpads of the ischemic rat hind limbs demonstrated ischemic lesions ranging from pressure sores to gangrene by 14 
A.

SMA+ Vessels in Ligated Limb

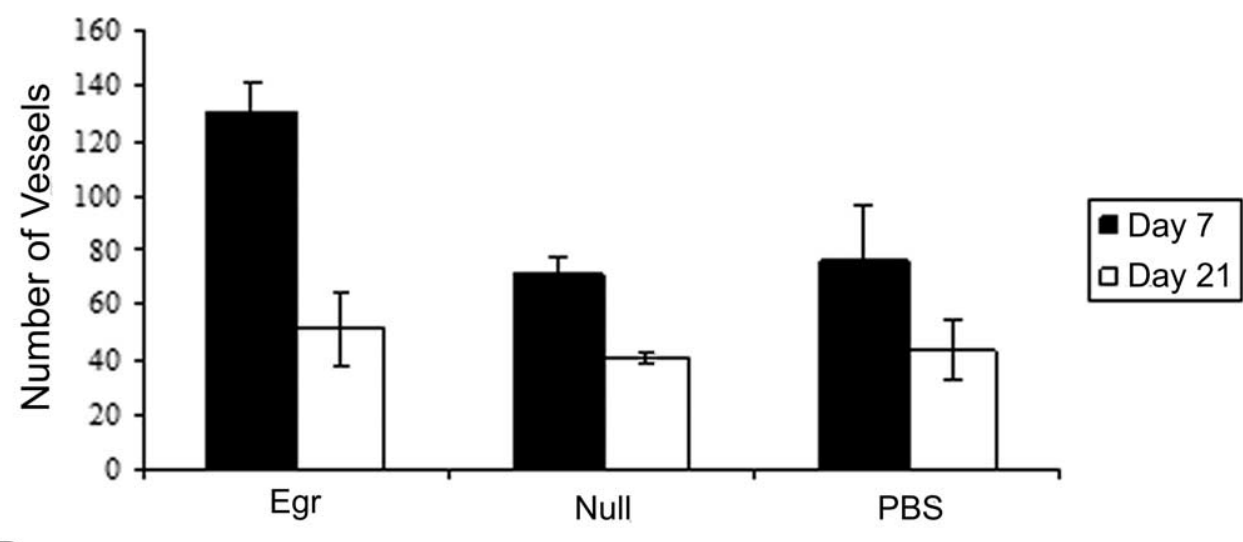

B.

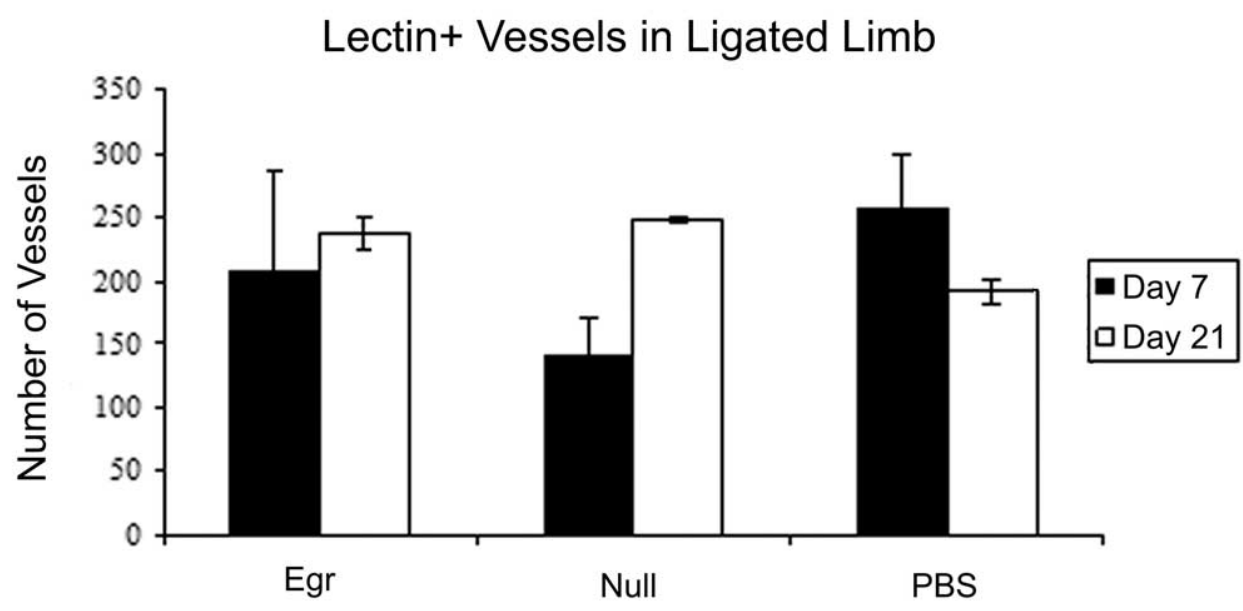

Figure 4. Density of arterioles and capillaries in ligated rat hind limb 21 days after specified treatments, as identified with smooth muscle cell actin (A) and lectin stains (B), respectively. SMA, Smooth muscle actin; Egr, early growth response gene (Ad transfer).

days after vascular excision. At 21 days after excision, the AdEgr-1-treated group demonstrated the least number of lesions in the most severe lesion classes (Figure 3) and less severe lesions overall compared with PBS- and AdNull-injected animals (mean ischemic score: $2.7 \pm 0.5$ vs $3.2 \pm 1.0$ and $4.1 \pm 0.9$, respectively; $P=.17$ ). Of note, gangrenous lesions (lesion score $4+$ or $5+$ ) were noted in none of the AdEgr-1-treated group compared with 7 (50\%) of the AdNulltreated animals or PBS-treated control animals, respectively $(P=.05)$.

\section{Histology}

AdEgr-treated rats demonstrated nearly twice as many SMA-positive vessels (arterioles) at day 7 after excision as did AdNull- or PBS-treated control animals (130 \pm 11 vs $70 \pm 6$ and $80 \pm 19$, respectively; $P<.001$ ). AdEgrtreated animals still demonstrated approximately $20 \%$ more SMA-positive vessels per microscopic field than did control animals by day 21 after excision, although the total number of such vessels had decreased in each group (Figure 4). The number of SMA-positive vessels measuring at least $50 \mu \mathrm{m}$ in diameter (arterioles) demonstrated a similar trend (day 7: $14 \pm 3$ for AdEgr-treated animals and $5 \pm 4$ for AdNull-treated animals, $P=.013$; day 21: $4 \pm 1$ for AdEgr-treated animals and $3 \pm 0$ for AdNulltreated animals, $P=.18$ ). In contrast, the total number of capillaries, as detected by means of lectin staining, was equivalent between groups at both the 7- and 21-day time points. The morphology of both large and small vessels was similar in AdEgr-treated and untreated animals (Figure 5). 

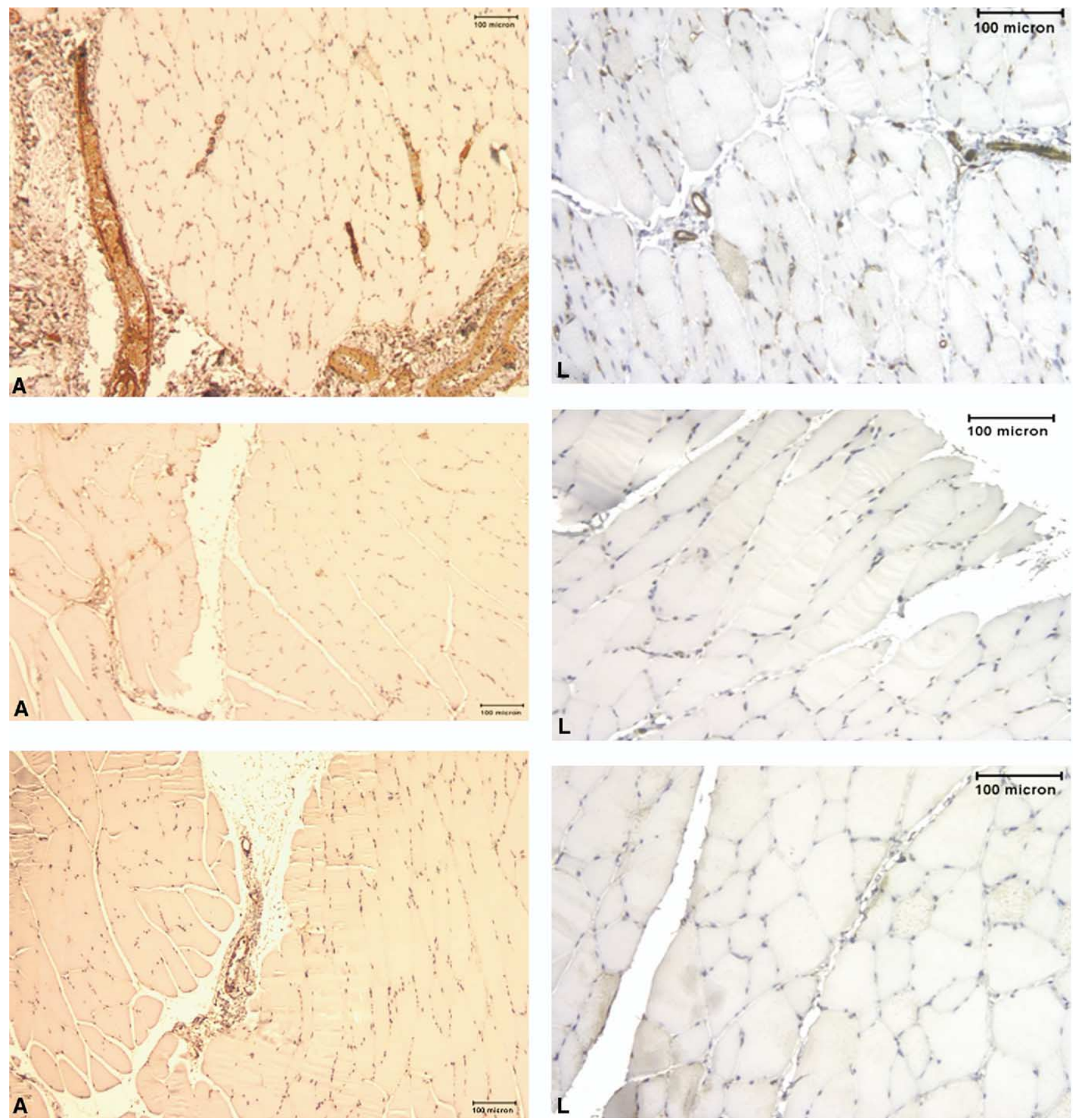

Figure 5. Photomicrographs of arterioles and capillaries in ligated rat hind limbs after specified treatments, as identified by smooth muscle cell actin (A) and lectin stains (L), respectively. Top panels, adenoviral vector encoding for Egr-1 (AdEgr); middle panels, phosphate-buffered saline (PBS); bottom panels, noncoding vectors (AdNull).

\section{Discussion}

A growing body of data suggest that Egr-1 plays an important role in a number of vascular homeostatic mechanisms, including those that might translate physiologic factors, such as shear stress and hypoxia, into modulation of gene expression and biologic responses, potentially including neovascularization after vascular occlusion. ${ }^{14-20,27-29}$ The apparent importance of
Egr-1 in a pneumonectomy-contralateral lung growth model that we have previously reported ${ }^{30}$ and the known biologic properties of Egr-1 that would be relevant to the hemodynamic changes expected with naturally occurring atherosclerotic vascular occlusions led us to suspect that Egr-1 might represent the previously missing signaling factor capable of translating the physiologic sequelae of vascular occlusion into the homeo- 
static response of collateral vessel formation that follows this biologic perturbation.

Our prior findings of essentially absent revascularization after vascular excision in an Egr-1 knockout mouse model supported our premise of the criticality of Egr-1 in postocclusive neovascularization processes. ${ }^{20}$ Consistent with these previous results, we now demonstrate a relative deficiency specifically in arteriogenesis in Egr-1 null versus wild-type mice and near-normalization of distal perfusion after AdEgr administration to ligated rats, apparently through a primarily arteriogenic process. These findings provide evidence that arteriogenesis is a critical mechanism underlying endogenous neovascularization processes and suggest a new means of providing for robust reperfusion in the treatment of atherosclerosis and vascular occlusive disease.

The present findings that Egr-1 deletion or administration appears primarily to affect the development of arterioles, rather than capillaries, are intriguing given the known molecular pathways governed by the Egr-1 transcription factor. More specifically, Egr-1 is known to upregulate the expression of genes, such as platelet-derived growth factor A and B and transforming growth factor $\beta$, implicated in smooth muscle cell recruitment and arteriogenesis and, indirectly through transforming growth factor $\beta$, upregulate a number of growth factors, including VEGF, and metalloproteinases that are responsible for angiogenesis and vascular formation. ${ }^{14-19} \mathrm{By}$ using smooth muscle cell actin staining as a marker of vascular smooth muscle recruitment and arteriolar development, ${ }^{31}$ the current findings suggest that although arteriogenesis might be closely linked to Egr-1 expression, angiogenesis might be mediated by pathways independent of Egr-1.

Much uncertainty surrounds the postnatal roles and relative physiologic importance of angiogenesis, the sprouting, bridging, and intussusceptive growth of capillaries from existing vessels, presumably facilitated by hypoxia, and arteriogenesis, the in situ enlargement of muscular collateral vessels from preexisting arteriolar anastomoses. ${ }^{8-13}$ It has been suggested that arteriogenesis, or collateral vessel development, occurring proximal to distal ischemic beds is most important for efficient revascularization and the rapid development of stable nurturing vessels that provide extensive blood flow and are not subject to regression in the setting of acute ischemia. ${ }^{32}$ In contrast, the capillary networks that rapidly develop in the setting of ischemia (angiogenesis) might undergo involution and are theoretically not ideal permanent conduits for a robust revascularization that are critical for providing nutrient and oxygen delivery to the distal ischemic tissues. ${ }^{32}$ Given the present histologic studies suggesting the primacy of arteriogenic processes after AdEgr administration, improved ischemic lesion scores in the present study suggest that an Egr-1-mediated pathway might provide for collateral development sufficient to enhance distal tissue bed perfusion. On the other hand, it is also possible that other trophic effects of Egr-1 or its downstream mediators, such as akt, are at least possibly responsible for the preservation of tissue integrity noted in the present studies, independent of the effects of Egr-1 on revascularization mechanisms.

Prior animal studies have shown that arteriogenesis involves rapid proliferation of preexisting arteriolar shunts, followed by remodeling processes. ${ }^{8-13,33,34}$ This process has a specific temporal dynamic, characterized by maximal proliferation in the first week after vascular occlusion, that has a biphasic progression that occurs independently of angiogenesis. Such considerations are consistent with the results of the current study.

Our finding that a primarily arteriogenic therapy can be used to enhance perfusion might be especially meaningful in that the near-normalization of distal perfusion mediated by AdEgr- 1 demonstrated in the current study exceeds results that we have previously obtained with the analogous delivery of angiogenic mediators, such as VEGF, in the described hind-limb excision model (data not shown). These findings suggest both the potency of arteriogenic pathways as mediators of physiologic perfusion and the potential efficacy of transcription factors, as opposed to the administration of individual growth factors, in such therapeutic revascularization strategies. ${ }^{34}$

\section{References}

1. Folkman J, Shing Y. Angiogenesis. J Biol Chem. 1992;267:10931-4.

2. Schaper W, Sharma HS, Quinkler W, Markert T, Wunsch M, Schaper J. Molecular biologic concepts of coronary anastomoses. J Am Coll Cardiol. 1990;15:513-8.

3. Carmeliet P. Angiogenesis in health and disease. Nat Med. 2003;9:653-60.

4. Epstein SE, Kornowski R, Fuchs S, Dvorak HF. Angiogenesis therapy: amidst the hype, the neglected potential for serious side effects. Circulation. 2001;104:115-9.

5. Takeshita S, Zheng LP, Brogi E, et al. Therapeutic angiogenesis. A single intraarterial bolus of vascular endothelial growth factor augments revascularization in a rabbit ischemic hind limb model. $J$ Clin Invest. 1994;93:662-70.

6. Freedman SB, Isner JM. Therapeutic angiogenesis for coronary artery disease. Ann Intern Med. 2002;136:54-71.

7. Simons M, Bonow RO, Chronos NA, et al. Clinical trials in coronary angiogenesis: issues, problems, consensus. An expert panel summary. Circulation. 2000;102:e73-86.

8. Ito WD, Arras M, Scholz D, et al. Angiogenesis but not collateral growth is associated with ischemia after femoral artery occlusion. 1997. Am J Physiol. 1997;42:H1255-65.

9. Diendl E, Buschmann I, Hoefer I, et al. Role of ischemia and of hypoxia-inducible genes in arteriogenesis after femoral artery occlusion in the rabbit. Circ Res. 2001;89:1-9.

10. Helisch A, Schaper W. Arteriogenesis: the development and growth of collateral vessels. Microcirculation. 2003;10:83-97.

11. Heil M, Clauss M, Suzuki K, et al. Vascular endothelial growth factor (VEGF) stimulates monocyte migration through endothelial monolayers via increased integrin expression. J Cell Biol. 2000;79:850-7.

12. Ito WD, Arras M, Winkler B, et al. Monocyte chemotactic protein-1 increases collateral and peripheral conductance after femoral artery occlusion. Circ Res. 1997;80:829-37. 
13. Carmeliet P. Mechanisms of angiogenesis and arteriogenesis. Nat Med. 2000;6:389-95.

14. Yan SF, Fujita T, Lu J, et al. Egr-1, a master switch coordinating upregulation of divergent gene families underlying ischemic stress. Nat Med. 2000;6:1355-61.

15. Gashler A, Sukhatme VP. Early growth response protein (EGR-1): prototype of a zinc-finger family of transcription factors. Prog Nucleic Acid Res Mol Biol. 1995;50:191-224.

16. Khachigian LM, Collins T. Inducible expression of Egr-1-dependent genes: a paradigm of transcriptional activation in vascular endothelium. Circ Res. 1997;81:457-61.

17. Silverman ES, Collins T. Pathways of Egr-1 mediated gene transcription in vascular biology. Am J Pathol. 1999;154:665-70.

18. Braddock M, Schwachtgen JL, Houston P, et al. Fluid shear stress modulation of gene expression in endothelial cells. Am J Physiol 1998;13: 241-6.

19. Mechtcheriakova D, Schabbaure G, Lucerna M, et al. Specificity, diversity, and convergence in VEGF and TNT- $\alpha$ signaling events leading to tissue factor up-regulation via Egr-1 in endothelial cells. FASEB J. 2001;15:230-42.

20. Schalch P, Patejunas G, Sarateanu CS, et al. Homozygous deletion of egr-1 results in critical limb ischemia following vascular ligation: evidence for a central role for EGR-1 in vascular homeostasis. $J$ Thorac Cardiovasc Surg. 2004;128:595-601.

21. Lee SL, Sadovsky Y, Swirnoff AH, et al. Luteinizing hormone deficiency and female infertility in mice lacking the transcription factor NGFI-A (Egr-1). Science. 1996;273:1219-21.

22. Tourtellotte WG, Nagarajan R, Auyeung A, et al. Infertility associated with incomplete spermatogenic arrest and oligozoospermia in Egr 4-deficient mice. Development. 1999;126:5061-71.

23. Hersh J, Crystal RG, Bewig BB. Modulation of gene expression after replication deficient recombinant adenovirus-mediated gene transfer by the product of a second adenovirus vector. Hum Gene Ther. 1995;2:12431.

24. Rosenfeld MA, Siegfried W, Yoshimura K, et al. In vivo transfer of the human cystic fibrosis transmembrane conductance regulator gene to the airway epithelium. Cell. 1992;68:143-55.

25. Couffinhal T, Sheng SM, Kearney M, et al. Mouse model of angiogenesis. Am J Pathol. 1998;152:1667-79.

26. Paek R, Chang DS, Brevetti DS, et al. Correlation of a simple direct measure of muscle $\mathrm{pO} 2$ to a clinical ischemia index and histology in a rat model of chronic severe hindlimb ischemia. J Vasc Surg. 2002;36:172-9.

27. McCaffrey TA, Fu C, Du B, et al. High-level expression of Egr-1 and Egr-1-inducible genes in mouse and human atherosclerosis. J Clin Invest. 2000;105:653-62.

28. Fahmy RC, Dass CR, Sun LQ, Chesterman CN, Khachigian LM. Transcription factor Egr- 1 supports FGF-dependent angiogenesis during neovascularization and tumor growth. Nat Med. 2003;9:1026-32.

29. Boengler K, Pipp F, Fernandez B, Ziegelhoeffer T, Schaper W, Deindl E. Arteriogenesis is associated with an induction of the cardiac ankyrin repeat protein (carp). Cardio Res. 2003;59:573-81.

30. Landesberg LJ, Ramalingam R, Lee K, et al. Upregulation of transcription factors in lung in the early phase of post-pneumonectomy lung growth. Am J Physiol. 2001;281:L1138-49.

31. Hughes S, Chan-Ling T. Characterization of smooth muscle cell and pericyte differentiation in the rat retina in vivo. Invest Ophthamol Vis Sci. 2004;45:2795-806.

32. Peirce SM, Skalak TC. Microvascular remodeling: a complex continuum spanning angiogenesis to arteriogenesis. Microcirculation. 2003; 10:1099-111.

33. Blau HM, Banfi A. The well-tempered vessel. Nat Med. 2001;7:532-4.

34. Hershey JC, Baskin EP, Glass JD, et al. Revascularization in the rabbit hindlimb: dissociation between capillary sprouting and arteriogenesis. Cardiovasc Res. 2001;49:618-25.

\section{Discussion}

Dr Frank W. Sellke (Boston, Mass). This study very elegantly and convincingly shows the importance of the early growth response 1 (Egr-1) gene in the development of collateral vessels and especially the role in the arteriogenesis process. However, there are many transcription factors and growth factors that have been examined that have demonstrated excellent results in providing increased blood flow to ischemic tissues in animal models. However, human trials have essentially been negative to date.

Do you think Egr-1 bypasses some of those endothelial-specific mediators or other mediators of angiogenesis and arteriogenesis that might be missing in patients with atherosclerosis, diabetes, hypertension, and other risk factors for coronary artery disease and a poor angiogenic response, or would this also be subject to those similar influences diminishing the angiogenic effect?

What is the role of shear forces in the regulation of Egr-1 expression? Is this one of the common pathways leading to the arteriogenesis process?

Finally, you showed an approximately $20 \%$ increase in perfusion at 21 days. Do you think this will be sufficient to provide enough blood flow to treat the ischemia?

Dr Rosengart. Thank you, Frank. Those are all very important questions.

In terms of the importance of Egr, it is certainly not completely clear at this point, but what we were most struck by is the absolute lack of reperfusion in our knockout animals, suggesting in fact that Egr might be at the top of the molecular pyramid governing revascularization, and that even though other mechanisms, such as ischemic upregulation of vascular endothelial growth factor, for example, should in theory be intact, in fact they did not seem to play a significant role in terms of revascularization. Therefore it is conceivable, although certainly not proved, with all the overlap in different molecular pathways, that Egr is certainly very important if not absolutely mandatory for revascularization.

Shear forces do play a very important role in Egr upregulation, and this is one of the things that attracted us to Egr as an interesting messenger. Certainly increases in shear forces that upregulate Egr, and is evidence as well that decreases in shear forces also play a role. Which of these forces is causing upregulation of Egr in this model is not clear, but we have certainly shown upregulated Egr in the vasculature after occlusion.

Finally, changes that we see in animal models, as you know and have so elegantly demonstrated, do not necessarily translate into the clinical scenario, and certainly this is an area that will need to be investigated.

Dr Michael Mann (San Francisco, Calif). I am sure you are familiar with the work of Wolfgang Schaper in Germany, who originally emphasized the role of these mechanical forces but has more recently looked at inflammation as a key mediator of the arteriogenesis response. Do you have any data that suggest exactly where the expression of Egr and the activity of Egr is essential? Is it actually in the endothelium or in the blood vessel? Is it active actually in your gene transfer model in the muscles, and what are the implications for potential hematherapies?

Dr Rosengart. Wolfgang Schaper is truly a pioneer in the area and has been an inspiration specifically in this work. If you look at the molecular pathways of Egr and monocyte chemotactic factor, for example, which is an area of tremendous interest for Professor Schaper, Egr is, in fact, upstream of monocyte chemotactic protein 1 and again, theoretically, is controlling in that manner. It might take precedence over inflammation, although certainly that would need to be looked at. We have demonstrated that Egr is upregulated within the vasculature wall-we have not isolated which cell type - which certainly makes sense in terms of Egr-1 being responsive to changes in shear stress. 